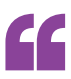

use of this

strategy eliminates the risk of graftversus-host disease

$\Rightarrow$ NEUROMETABOLIC DISEASE

\title{
Gene therapy success for cerebral adrenoleukodystrophy
}

Cerebral adrenoleukodystrophy (ALD) could be treated safely and effectively using genetically modified versions of a patient's own haematopoietic stem cells, according to a new study. The gene therapy offers an alternative to allogeneic stem cell transplantation, which is often associated with a high risk of adverse immunological events.

ALD is an X-linked disease caused by mutations in $A B C D 1$, which result in a peroxisomal metabolic disorder characterized by defective breakdown of fatty acids. Often, the disease progresses to cerebral inflammation and rapid demyelination. Cerebral ALD primarily affects boys $<12$ years of age and leads to death or severe disability within 2 years if untreated.

Currently, the only known effective treatment for cerebral ALD is allogeneic haematopoietic stem cell transplantation. However, identification of donors with a complete antigenic match is problematic for many patients, and a poor match is associated with a high risk of adverse immunological events such as graft failure and graft-versus-host disease.

In the new phase II-III

STARBEAM trial, investigators recruited 17 boys (aged $<18$ years) with cerebral ALD. The team isolated haematopoietic stem cells from the patients and transfected them with a lentiviral vector containing a functional cDNA copy of $A B C D 1$. The researchers then examined whether transplantation of these modified cells - a therapy termed Lenti-D (bluebird bio) - back into the patients was safe and effective.

Of 17 patients, 15 met the primary study end point of survival with no major functional disabilities at 24 months. Two patients died of disease-related causes that were unrelated to the treatment. In addition, 14 of 17 patients showed no or minimal clinical symptoms, and 12 of 17 patients exhibited stabilization of lesion progression on MRI.

The findings suggest that this therapy is at least as safe as existing allogeneic transplantation strategies.
Importantly, use of this strategy eliminates the risk of graft-versushost disease and could save valuable time in patient treatment that would otherwise be spent finding a suitable donor, thereby avoiding accumulation of additional white matter impairments. However, the authors note that a longer follow-up period and larger sample size are needed to verify the efficacy and safety of their approach.

"For many years, gene therapy has shown great promise, but clinical applications have always seemed just beyond the horizon," comments Marc Engelen in an editorial accompanying the new paper. "Today, Lenti-D therapy appears to be poised as a real treatment option for cerebral adrenoleukodystrophy, and it might even become the first gene therapy approved by the FDA."

Charlotte Ridler

ORIGINAL ARTICLE Eichler, F. et al.

Hematopoietic stem-cell gene therapy for cerebral adrenoleukodystrophy. N. Engl.J. Med. http://dx. doi.org/10.1056/NEJMoa1700554 (2017) 